\title{
QUATERNION WAVELETS FOR IMAGE ANALYSIS AND PROCESSING
}

\author{
Wai Lam Chan, Hyeokho Choi, Richard Baraniuk \\ Department of Electrical and Computer Engineering, Rice University \\ Houston, Texas, USA
}

\begin{abstract}
Using the concepts of two-dimensional Hilbert transform and analytic signal, we construct a new quaternion wavelet transform (QWT). The QWT forms a tight frame and can be efficiently computed using a 2-D dual-tree filter bank. The QWT and the 2-D complex wavelet transform (CWT) are related by a unitary transformation, but the former inherits the quaternion Fourier transform (QFT) phase properties, which are desirable for image analysis. The quaternion magnitude-phase representation of the QWT directly leads to near shift-invariance and the ability to encode phase shifts in an absolute $x y$-coordinate system, which we can use for applications such as edge estimation and statistical image modeling.
\end{abstract}

\section{INTRODUCTION}

Wavelet transforms are powerful multiscale tools for processing singularity rich signals. Unlike the Fourier basis functions, the locality of wavelet basis functions leads to sparse representation of singularity rich signals by compacting the signal energy into a small number of coefficients. Wavelet coefficient sparsity is critical to algorithms such as wavelet signal denoising by shrinkage [1]. In two-dimensions (2-D), because natural images typically consist of wide smooth regions separated by edges, the supremacy of wavelet transforms is even more evident. Conventional 2-D wavelet basis functions are obtained by simple Cartesian product of I-D wavelet functions.

However, one of the major problems of real-valued wavelets is their lack of shift-invariance. A small shift of the signal results in significant fluctuations of wavelet coefficient energy, making it difficult to extract signal information from the coefficient values. The undecimated wavelet transform [2] provides a shift invariant transform, but at the cost of high redundancy.

To remedy the problem of shift variance, there have been significant research efforts to develop nearly shift-invariant wavelet transforms with moderate redundancy. The complex wavelet transform (CWT) uses complex wavelet basis functions whose real and imaginary parts are 1-D Hilbert transform pairs $[3,4]$. Because the real and imaginary parts are in quadrature, the CWT coefficient magnitudes are almost shift invariant with moderate redundancy $[3,4]$.

In this paper, we leverage the notions of 2-D Hilbert transform and 2-D analytic signal to build a new 2-D shift-invariant wavelet transform. We adopt the definition of 2-D Hilbert transform in [5]

Email: \{wailam, choi, richb\}@rice.edu. Web; dsp.rice.edu. This work was supported by ONR grant N00014-02-1-0353, AFOSR grant FA9550-04-1-0148, DARPA, and the Texas Instruments Leadership University Program. by Bülow and Sommer to build a new quaternion wavelet transform (QWT). The 2-D Hilbert transforms of a 2-D DWT tensor product wavelet (i.e., the I-D Hilbert transform of the wavelet along either or both of the horizontal and vertical directions) plus the wavelet itself constitute the four components of a quaternion wavelet. The QWT frame, which can be efficiently generated from a dual-tree filter bank [4], is a $4 \times$ redundant tight frame and therefore is stably invertible.

Besides shift-invariance, another advantage of the QWT for image analysis is that it encodes image shifts in an absolute $x y$ coordinate system. These properties give us insights into building a QWT hidden Markov tree (HMT) model [6] for images that captures both persistency of magnitudes and coherence of phase across scale. We can also relate the QWT to the 2-D CWT in $[4,7]$ through a unitary transformation. In image analysis, the 2-D CWT is well suited for 1-D features (edges) [8] while, inherited from the QFT [5], the QWT is particularly suitable for analyzing 2-D features (textures). Beyond 2-D, signals typically contain more complicated low dimensional manifold structures. The generalization of the Hilbert transform to $n$-D using hypercomplex numbers can be used to develop wavelet transforms in higher dimensions suitable for these signals [9].

This paper is organized as follows. In Sections 2 and 3 we briefly review the DWT and the CWT. Section 4 develops the QWT and Section 5 discusses some important properties of the new transform. Section 6 summarizes the paper and emphasizes the importance of our contributions.

\section{DISCRETE WAVELET TRANSFORM (DWT)}

The discrete wavelet transform (DWT) represents a 1-D real signal $f(t)$ in terms of shifted versions of a scaling function $\phi(t)$ and shifted and scaled versions of a wavelet function $\psi(t)$ [10]. When $\phi_{L, p}(t)=2^{L} \phi\left(2^{L} t-p\right)$ and $\psi_{\ell, p}(t)=2^{\ell} \psi\left(2^{\ell} t-p\right), \ell \geq L, p \in$ $\mathbb{Z}$, form an orthonormal basis, we can represent any $f(t) \in L_{2}$ as

$$
f(t)=\sum_{p \in \mathbb{Z}} c_{L, p} \phi_{L, p}(t)+\sum_{\ell \geq L, p \in \mathbb{Z}} d_{\ell, p} \psi_{\ell, p}(t),
$$

where $c_{L, p}=\int f(t) \phi_{L, p}(t) d t$ and $d_{\ell, p}=\int f(t) \psi_{\ell, p}(t) d t$ are the scaling and wavelet coefficients. $L$ sets the coarsest scale space that is spanned by $\phi_{L, p}(t)$. Behind each wavelet transform is a filterbank based on a lowpass filter: we will use the notation $\phi_{k}(t)$, $\psi_{h}(t)$ to denote the scaling and wavelet functions corresponding to a particular filter $h$.

The 2-D DWT is obtained using tensor products of I-D DWTs over each dimension: the scaling function $\phi(x) \phi(y)$ and three wavelets $\psi(x) \psi(y), \phi(x) \psi(y)$, and $\psi(x) \phi(y)$ are oriented in the diagonal, horizontal, and vertical directions, respectively [7]. 


\section{COMPLEX WAVELET TRANSFORM (CWT)}

The 1-D dual-tree CWT expands a real signal in terms of two sets of wavelet and scaling functions obtained from two independent filterbanks [4]. $\psi_{h}(t)$ and $\psi_{g}(t)$ play the role of the real and imaginary parts of a complex analytic wavelet $\psi^{c}(t)=\psi_{h}(t)+j \psi_{g}(t)$. The imaginary wavelet is the 1-D Hilbert transform of the real wavelet. The combined system is a $2 \times$ redundant frame that, by virtue of the fact that $\left|\psi^{c}(t)\right|$ is non-oscillatory, is shift-invariant. ${ }^{1}$

It is useful to recall that the Fourier transforms of the Hilbert pair of wavelets are related by $\Psi_{g}(\omega)$, which equals $-j \Psi_{h}(\omega)$, when $\omega>0$ and $j \Psi_{h}(\omega)$ when $\omega<0$. Thus, summing $\Psi_{h}(\omega)+$ $j \Psi_{g}(\omega)=\Psi^{c}(\omega)$ cancels the negative frequency part of the complex wavelet. rendering it analytic.

To develop a near shift-invariant wavelet transform in 2-D, we thus turn to the theory of 2-D Hilbert transforms and 2-D analytic signals.

\section{QUATERNION WAVELET TRANSFORM (QWT)}

There is no unique definition of the Hilbert transform and analytic signal in 2-D and higher. To illustrate two possibilities, consider the Fourier transform of a 2-D wavelet $\Psi(u, v)$. The straightforward extension of the 1-D definition cancels out frequencies on all but a half-plane of the Fourier space ( $u>0$, for example) and uses standard complex algebra for manipulation. A second natural definition cancels out frequencies on all but a quadrant $(u, v>0$. for example) and uses quaternion algebra for manipulation [5]. ${ }^{2}$

Adopting the latter definition [5], we now introduce a new multiscale signal representation: the dual-tree quaternion wavelet transform (QWT).

Each quaternion wavelet consists of a standard DWT tensor wavelet plus three additional real wavelets obtained by 1-D Hilbert transforms along either or both coordinates. More specifically, we denote the 1-D Hilbert transform operators along the $x$ and $y$ coordinates by $\mathcal{H}_{x}$ and $\mathcal{H}_{y}$, respectively. Then given the usual real tensor product wavelet $\psi_{h}(x) \psi_{h}(y)$ from Section 2, we complement it with

$$
\begin{aligned}
\mathcal{H}_{x}\left\{\psi_{h}(x) \psi_{h}(y)\right\} & =\psi_{g}(x) \psi_{h}(y) \\
\mathcal{H}_{y}\left\{\psi_{h}(x) \psi_{h}(y)\right\} & =\psi_{h}(x) \psi_{g}(y) \\
\mathcal{H}_{y} \mathcal{H}_{x}\left\{\psi_{h}(x) \psi_{h}(y)\right\} & =\psi_{g}(x) \psi_{g}(y)
\end{aligned}
$$

Conveniently, each component can be computed as a combination of 1-D dual-tree complex wavelets. These three components resemble $\psi_{h}(x) \psi_{h}(y)$ but are phase-shifted by $90^{\circ}$ in the horizontal, vertical, and both directions, respectively. We can interpret these relationships in the Fourier domain as multiplying the quadrants of the Fourier transform of $\psi_{h}(x) \psi_{h}(y)$ by $\pm j$ (where $j=\sqrt{-1}$ ) and \pm 1 as shown in Fig. 1.

Using quaternion algebra, we can organize the four wavelet functions above into a quaternion wavelet $\psi^{q}(x, y)=\psi_{h}(x) \psi_{h}(y)$ $-j_{1} \psi_{g}(x) \psi_{h}(y)-j_{2} \psi_{h}(x) \psi_{g}(y)+j_{3} \psi_{g}(x) \psi_{g}(y)$. Figure 2 shows the 4 components of a quaternion wavelet. One can observe the $90^{\circ}$ phase shift of the components relative to each other. The magnitude-square of the quaternion basis $\left|\psi^{q}(x, y)\right|^{2}$ (sumof-squares of all four components) is non-oscillatory, indicating

\footnotetext{
'In practice, in order to have finite-length wavelets, the Hilbert transform is only approximately satisfied, $\psi^{c}(t)$ is only approximately analytic, and the CWT is only approximately shift-invariant $[4,7]$.

2The set of quatemions $\mathbb{H}=\left\{a+j_{1} b+j_{2} c+\left.j_{3} d\right|_{a}, b, c, d \in \mathbb{R}\right\}$ with multiplication rules $j_{1} j_{2}=-j_{2} j_{1}=j_{3}$ and $j_{1}^{2}=j_{2}^{2}=-1$ [11].
}

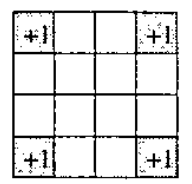

(a) $\Psi_{h}(u) \Psi_{h}(v)$

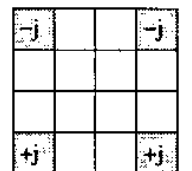

(c) $\Psi_{h}(u) \Psi_{g}(v)$

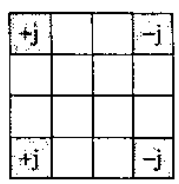

(b) $\Psi_{g}(u) \Psi_{h}(v)$

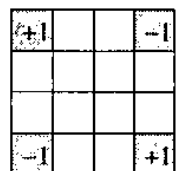

(d) $\Psi_{g}(u) \Psi_{3}(v)$
Fig. 1. Fourier-domain relationships among the four components of a $Q W T$ wavelet $\Psi(u, v)$ in the diagonal subband.

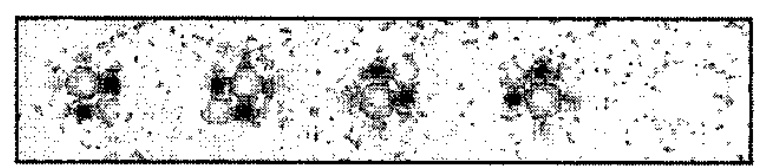

Fig. 2. Each quatemion wavelet basis contains 4 components. Shown above is the set from the diagonal subband, from left to right: $\psi_{h}(x) \psi_{h}(y), \psi_{h}(x) \psi_{g}(y), \psi_{g}(x) \psi_{h}(y), \psi_{g}(x) \psi_{g}(y)$. The final image is $\left|\psi^{q}(x, y)\right|$, a non-oscillatory function, which implies the shift-invariance of the QWT [3, 4].

that the transform is approximately shift-invariant. The construction and properties are similar for the other two quaternion wavelets based on $\phi_{h}(x) \psi_{h}(y)$ and $\psi_{h}(x) \phi_{h}(y)$.

Our quaternion wavelet transform is similar to the quaternion Fourier transform (QFT) in the use of quaternion algebra [5]. The 2-D quaternion wavelets, like the 2-D analytic signal described in [5]. have spectral support only in a single (upper-right) quadrant in the QFT domain. The QFT of a real 2-D signal $f$ is

$$
F^{q}(\mathbf{u})=\int_{\mathbb{R}^{2}} e^{-j_{1} 2 \pi u x} f(\mathbf{x}) e^{-j_{2} 2 \pi v y} d \mathbf{x},
$$

where $\mathbf{u}=(u, v), \mathbf{x}=(x, y)$, and the quaternion exponentials $e^{-j_{1} 2 \pi u x} e^{-j_{2} 2 \pi v y}$ are the basis elements. The basis elements for the QWT are the quaternion wavelets $\left(\psi(x)-j_{1} \psi(y)\right)(\psi(x)-$ $\left.j_{2} \psi(y)\right)$ plus the two wavelets in the other subbands with various scales and translations. Therefore, the QWT inherits some of the useful properties from the QFT.

\section{PROPERTIES}

In terms of efficient implementation and image processing applications, the QWT enjoys some of the advantageous properties of both the 2-D CWT and QFT.

\subsection{Tight frame}

The QWT, which contains four orthonormal basis sets. forms a $4 \times$ redundant tight frame [10]. The QWT wavelet functions can be written in a matrix form

$$
G=\left[\begin{array}{lll}
\psi_{h}(x) \psi_{h}(y) & \psi_{h}(x) \phi_{h}(y) & \phi_{h}(x) \psi_{h}(y) \\
\psi_{g}(x) \psi_{h}(y) & \psi_{g}(x) \phi_{h}(y) & \phi_{g}(x) \psi_{h}(y) \\
\psi_{h}(x) \psi_{g}(y) & \psi_{h}(x) \phi_{g}(y) & \phi_{h}(x) \psi_{g}(y) \\
\psi_{g}(x) \psi_{g}(y) & \psi_{g}(x) \phi_{g}(y) & \phi_{g}(x) \psi_{g}(y)
\end{array}\right]
$$

Each column of the matrix $G$ in (6) contains the 4 components of the quaternion wavelet corresponding to a subband of the QWT. For example, the first column contains the quaternion 
wavelet components in Fig. 2. i.e., the tensor product wavelet $\psi_{h}(x) \psi_{h}(y)$ and its 2-D Hilbert transforms in equations (2)-(4) in Section 4.

On the other hand. each row of $G$ contains the wavelet functions necessary (with all combinations of scales and shifts) to form one orthonormal basis set. Since $G$ has four rows, the quaternion wavelets form a $4 \times$ redundant tight frame, and thus is stably invertible. All wavelet bases in $G$ can be generated using a 2-D dual-tree filter bank with $O(N)$ efficiency.

\subsection{Relationship to CWT}

The 2-D CWT is a near shift-invariant wavelet frame, with basis functions oriented along six 1-D directions [4, 7]. These basis functions are obtained by the multiplication of two 1-D complex wavelets, $\psi^{c}(x) \psi^{c}(y)$, on the 2-D plane [7]. For instance, the formula for the complex wavelet oriented at $45^{\circ}$ is $\left(\psi_{h}(x) \psi_{h}(y)\right.$ $\left.\psi_{g}(x) \psi_{g}(y)\right)+j\left(\psi_{h}(x) \psi_{g}(y)+\psi_{g}(x) \psi_{h}(y)\right)$. Figure 3 shows the spectral support of these complex wavelet functions.

There exists a unitary transformation between the QWT and the 2-D CWT. We can obtain the CWT wavelet functions by multiplying matrix $G$ in (6) with a unitary matrix

$$
A=\frac{1}{\sqrt{2}}\left[\begin{array}{cccc}
1 & 0 & 0 & 1 \\
0 & 1 & 1 & 0 \\
0 & 1 & -1 & 0 \\
1 & 0 & 0 & -1
\end{array}\right]
$$

Therefore, both the CWT and the QWT are tight frames with $4 \times$ redundancy. As described in the next section. the phases of both the CWT and the QWT encode 2-D signal shifts. However. there exists no straightforward relationship between the QWT and the CWT phase angles.

\subsection{Quaternion phase angles}

Using quaternion algebra, we can express each quaternion wavelet coefficient as $q=|q| e^{j_{1} \theta_{1}} e^{j_{3} \theta_{3}} e^{j_{2} \theta_{2}}$ [5], where $|q|$ is the modulus of $q$ and $\left(\theta_{1}, \theta_{2}, \theta_{3}\right)$ are the phase angles of $q$, with each angle uniquely defined within intervals: $\left(\theta_{1}, \theta_{2}, \theta_{3}\right) \in[-\pi, \pi) \times$ $\left[-\frac{\pi}{2}, \frac{\pi}{2}\right) \times\left[-\frac{\pi}{4}, \frac{\pi}{4}\right]$. For an image block with QWT coefficient $q$. $|q|$ is almost invariant to signal shift and $\left(\theta_{1}, \theta_{2}\right)$ encode the shift.

The shift theorem for the QFT [5] approximately holds for the QWT because the QWT conducts a local QFT analysis. The theorem says, when a shift of image $f(\mathbf{x})$ to $f(\mathbf{x}-\mathbf{d})$ occurs, the QFT phase undergoes the following changes: $\left(\theta_{1}(\mathbf{u}), \theta_{2}(\mathbf{u}), \theta_{3}(\mathbf{u})\right) \rightarrow$ $\left(\theta_{1}(\mathbf{u})-2 \pi u d_{1}, \theta_{2}(\mathbf{u})-2 \pi v d_{2}, \theta_{3}(\mathbf{u})\right)$ where $\mathbf{u}=(u, v)$ are the axes of the 2-D QFT domain and $\mathbf{d}=\left(d_{\mathfrak{i}}, d_{2}\right)$. In the context of the QWT, $(u, v)$ is the effective center of the signal spectrum within the frequency tile of the corresponding QWT basis for each coefficient. Therefore, $(u, v)$ depends on the subband and scale of QWT basis and the actual shape of the signal spectrum.

Figure 4 contrasts the phase property of the QWT to the CWT. The image blocks in Fig. 4(a) and (b) undergo the same amount of shift toward the top left corner. Figure 4(a) illustrates how each CWT coefficient encodes the signal shift $d$ along the direction perpendicular to the corresponding wavelet (e.g., $-45^{\circ}$ wavelet as shown), since it has only one phase angle. On the other hand. the QWT encodes signal shift $\left(d_{1}, d_{2}\right)$ in $x y$-coordinates using two phase angles $\left(\theta_{1}, \theta_{2}\right)$ as in Fig. 4(b).

Based on the shift theorem. we can estimate the image shift $\left(d_{1}, d_{2}\right)$ from the phase shift $\left(\Delta \theta_{1}, \Delta \theta_{2}\right)$ relative to a reference image. Conversely, we can estimate the phase shift once we know the signal shift. However. we need to first estimate the effective spectral center $(\widehat{u}, \widehat{v})$ for each QWT coefficient. For images having

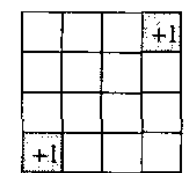

(a) $\mathscr{F}\left(\operatorname{Re}\left(\psi^{c}(x, y)\right)\right)$

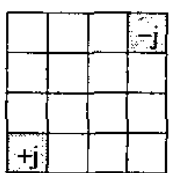

(b) $\mathscr{F}\left(\operatorname{Im}\left(\psi^{c}(x, y)\right)\right)$
Fig. 3. Fourier-domain relationships between the real and imaginary parts of a 2-D diagonally oriented complex wavelet. $\mathscr{F}(\cdot)$ is the 2-D Fourier Transform.

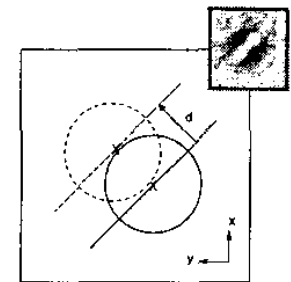

(a)

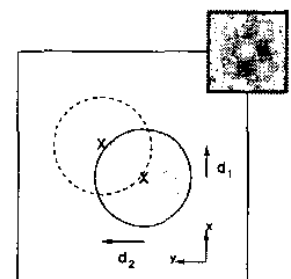

(b)
Fig. 4. (a) The CWT has one phase angle linear in image shift $d$ orthogonal to the wavelet's orientation. (b) The QWT has two phase angles to encode shifts, each linear in image shift $\left(d_{1}, d_{2}\right)$ respectively in absolute $x y$-coordinates.

smooth spectrum over the local spectral tile corresponding to each QWT basis, the center of the tile can be used as $(\widehat{u}, \widehat{v})$. Note that $(\widehat{u}, \widehat{v})$ should always lie in the first quadrant of the QFT domain (i.e., $\widehat{u}, \widehat{v}>0$ ).

When the image block contains a single edge as illustrated in Fig. 5(a), the spectrum is not smooth and we cannot choose $(\widehat{u}, \widehat{v})$ to be the center of the local spectral tile. The QFT spectrum of a single edge image in Fig. 5(a) lies on two lines through the origin having orientations $90^{\circ}-\beta$ and $\beta-90^{\circ}$ in the QFT domain. Therefore, the effective spectral center can be expressed as $(\widehat{u}, \widehat{v})=c(|\cos \beta|,|\sin \beta|)$ where $c$ is a positive constant that depends on $\beta$ and the location of the spectral tile corresponding to each QWT basis.

For an edge oriented at angle $\beta$, any shift $\left(d_{1}, d_{2}\right)$ in the $(x, y)$ directions satisfying the constraint

$$
d_{1} \cos \beta+d_{2} \sin \beta=d
$$

corresponds to the same shift as shifting the edge by $d$ perpendicularly. Using the relations $(\widehat{u}, \widehat{v})=c(|\cos \beta|,|\sin \beta|)$ in (8) and noting that $2 \pi \widehat{u} d_{1}=\Delta \theta_{1}$ and $2 \pi \widehat{v} d_{2}=\Delta \theta_{2}$, we obtain the expression

$$
d=\frac{\Delta \theta_{1} \pm \Delta \theta_{2}}{2 \pi c}
$$

where we choose $\Delta \theta_{1}+\Delta \theta_{2}$ when $\tan \beta>0$, and $\Delta \theta_{1}-\Delta \theta_{2}$ when $\tan \beta<0$.

To verify this relationship, we apply the QWT to the edge image in Fig. 5(a) and analyze the QWT magnitudes and phases corresponding to a $32 \times 32$ sub-block. Fig. 5 (b) and (c) verify that the coefficient magnitudes are almost invariant to signal shift $d$. Note that the magnitude is maximum when the edge orientation matches the 1-D direction of the basis function $\left(0^{\circ}\right.$ for $\phi(x) \psi(y)$ and $45^{\circ}$ for $\psi(x) \psi(y)$ subband). We also observe a linear relationship between $\Delta \theta_{1} \pm \Delta \theta_{2}$ and $d$ for various $\beta$ in all subbands. Using the slopes of these linear graphs, we can first estimate $c$, and then estimate the edge offset $d$ from the QWT phase using $d=0$ as the reference image. For algorithmic simplicity, we obtain an estimate of $c \approx 0.7$ for the $\psi(x) \phi(y)$ subband using the vertical edge $(\beta=0)$ which gives the largest QWT magnitude for this 


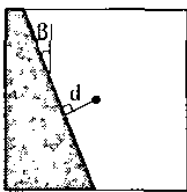

(a) Edge image

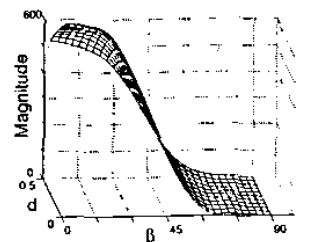

(b) Magnitudes

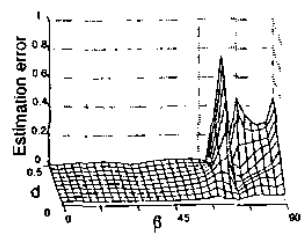

(d) Estimation crrors

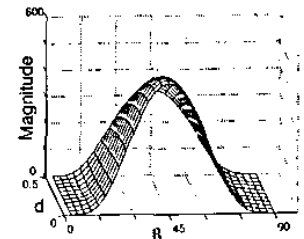

(c) Magnitudes

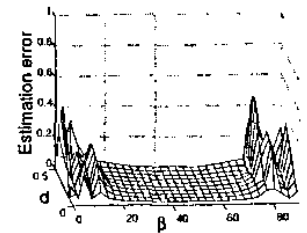

(e) Estimation errors
Fig. 5. (a) Parametrization of an edge in a dyadic block (side length $=1$ ). (b) and (c) show the magnitudes of QWT coefficient as a function of edge orientation $\beta$ and offset $d$ for the $\phi(x) \psi(y)$ and $\psi(x) \psi(y)$ subbands respectively. (d) and (e) show the absolute error for estimation of $d$ by phase angles $\left(\theta_{1}, \theta_{2}\right)$. Estimates are accurate in the region where coefficient magnitudes are large.

subband: similarly for the $\psi(x) \phi(y)$ subband. Using the $45^{\circ}$ edge. we obtain an estimate of $c \approx 1$ for the $\psi(x) \psi(y)$ subband. This simplification of using the same $c$ in each subband to estimate $d$ is valid because $c$ is more sensitive to the location of the spectral tile of the corresponding QWT basis than to $\beta$. Shown in Fig. 5(d) and (e) are the estimation results for 2 subbands across various $\beta$ and $d$. In small-magnitude regions, the phase angles are very sensitive to noise, and thus estimation errors are large. Using only the phase change of the QWT subband with the largest magnitude, we can estimate $d$ with a maximum error of $2 \%$.

Besides edge shift estimation. the shift-invariance and phase properties also make the QWT a convenient tool for constructing statistical image models. Wavelet transforms, due to their multiscale nature, have been successful in modeling piecewise smooth images. For example, Romberg $e t$ al. use a Hidden Markov Tree (HMT) model to exploit dependencies of complex wavelet coefficients across scale [6]. Natural images are composed of either smooth regions or single edge regions at finer scale. The magnitudes of wavelet coefficients are significant only in the edge regions. In these regions, there are two important observations. First, if the parent coefficient magnitude is large, then its child is also large if it also contains the edge. The QWT can capture this persistency of magnitudes across scale well because of its almost shiftinvariant nature. Second. the edge in the child has the same orientation as the parent but a different offset (i.e., phase). Based on our experiments with the single-edge image in Fig. 5(a), we can estimate the child phase from the parent phase, either deterministically from the experimental data (phase across $\beta$ and $d$ ) or statistically through algorithms derived from the mapping between $\left(\Delta \theta_{1}, \Delta \theta_{2}\right)$ and $(\beta . d)$. Such a model could greatly improve the de-

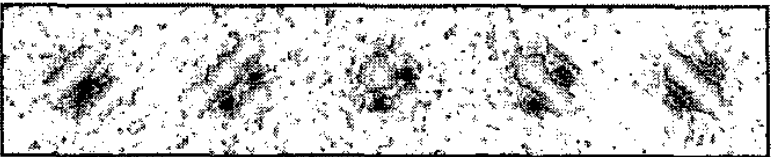

Fig. 6. Varying $\theta_{3}$ of a $Q W T$ coefficient from the diagonal subband (left to right): $\theta_{3}=-\frac{\pi}{4},-\frac{\pi}{8}, 0, \frac{\pi}{8}, \frac{\pi}{4}$ respectively. Corresponding wavelet changes from texture-like structure $\left(\theta_{3}=0\right)$ to edge-like structure $\left(\theta_{3}= \pm \frac{\pi}{4}\right)$.

sign of image processing tools for applications such as denoising and classification.

Finally, we interpret the third QWT phase angle $\theta_{3}$ as the relative amplitude of signal energy along the 1-D manifolds in two orthogonal directions as in [5]. By adjusting only $\theta_{3}$ of the QWT $\psi(x) \psi(y)$ subband coefficients, we can observe a gradual change in the appearance of the wavelet basis from directed to texture-like and back (see Fig. 6). This property of the QWT will prove useful for the analysis of images with rich textures [5].

\section{CONCLUSIONS}

In this paper, we have developed the theory of the dual-tree quaternion wavelet transform (QWT) using the concepts of 2-D Hilbert transforms and 2-D analytic signals. The QWT enjoys desirable properties such as near shift-invariance, invertibility and, in particular, the ability to encode phase shifts in an absolute coordinate system. The shift theorem, inherited from its quaternion Fourier counterpart. enables us to estimate edge offset efficiently and incorporate the QWT phase into an existing HMT image model. By generalizing the Hilbert transform to $n-\mathrm{D}$, we can obtain a hypercomplex wavelet transform, which is suitable for the analysis of signals with more complicated manifold structures [9].

\section{REFERENCES}

[1] D. L. Donoho, "De-noising by soft-thresholding," IEEE Truns. Info. Theory, vol. 41, no. 3, pp. 613-627, 1995.

[2] M. Lang, H. Guo, J. Odegard, C. Burrus, and R. Wells, "Noise reduction using an undecimated discrete wavelet transform." IEEE Signal Processing Letters, April 1995.

[3] J. M. Lina and M. Mayrand, "Complex Daubechies wavelets," ACHA, vol. 2, pp. 219-229, 1995.

[4] N. G. Kingsbury, "Complex wavelets for shift invariant analysis and filtering of signals," $A C H A$, vol. 10, no. 3, pp. 234-253, May 2002.

[5] T. Bülow, M. Felsberg, and G. Sommer, Geometric Computing with Clifford Algebras, Springer-Verlag, Berlin, 2001.

[6] J. Romberg, H. Choi, and R. Baraniuk, "Multiscale edge grammars for complex wavelet transforms," in Proc. of ICIP. Thessaloniki, Greece, October 2001.

[7] I. W. Selensnick and K. Y. Li, "Video denoising using 2-d and 3-d dual-tree complex wavelet transforms," in Proc. of SPIE Wavelets $X$, San Diego, CA, August 4-8 2003, vol. 76.

[8] J. Romberg, M. Wakin, H. Choi, and R. Baraniuk, "A geometric hidden Markov tree wavelet model," in Proc. of SPIE Wavelets X, San Diego, CA, August 2003.

[9] W. L. Chan, H. Choi, and R. Baraniuk, "Directional hypercomplex wavelets for multidimensional signal analysis and processing," in Proc. of ICASSP, Montreal, Canada, May 2004.

[10] M. Vetterli and J. Kovacevic, Wavelets and Subband Coding, Prentice Hall, Englewood Cliffs, NJ, 1995.

[11] I. L. Kantor and A. S. Solodovnikov, Hypercomplex Numbers, Springer-Verlag, 1989. 